FACTA UNIVERSITATIS (NIŠ)

Ser. Math. Inform. Vol. 35, No 2 (2020), 423-435

https://doi.org/10.22190/FUMI2002423S

\title{
ON BÄCKLUND TRANSFORMATIONS WITH SPLIT QUATERNIONS
}

\author{
Muhammed Talat Sariaydin
}

(C) 2020 by University of Niš, Serbia | Creative Commons Licence: CC BY-NC-ND

\begin{abstract}
The present paper deals with the introduction of Bäcklund transformations with split quaternions in Minkowski space. Firstly, we have briefly summarized the basic concepts of split quaternion theory and Bishop frames of non-null curves in Minkowski space. Then, for Bäcklund transformations defined with each case of non-null curves, we have given the relationships between Bäcklund transformations and split quaternions. Some special propositions for transformations constructed with split quaternions have also been presented. At the end, the results obtained with the mathematical model have been evaluated.
\end{abstract}

Keywords: Minkowski space; quaternions; Bäcklund transformations; Bishop frames.

\section{Introduction}

Bäcklund transformations give a correlation between PDE and their solution. In other words, one can estimate Bäcklund transformations generating a PDEs's solution if we know a solution of PDE. There exists a class of Bäcklund transformations which are called auto-Bäcklund transformations, when the connected PDEs are the same. To generate new solutions on the integrable theories the Bäcklund transformations have been widely used. These transformations help to connect diffucult PDE to simpler one that has easier solution. In the case of solutions, these transformations are highly effective in generating multi-solutions from the familiar solutions. By applying Bäcklund transformations to trivial solution one can generate a non-trivial case [16]. Due to the aforementioned features, numerous studies have been carried out on Bäcklund transformations from past to present. For example, Weiss studied the Bäcklund transformations on focal surfaces in [14], Sen gave darboux Bäcklund transformation of nonlinear optical waves in [12], B $\ddot{a}$ ck obtained Bäcklund transformations for minimal surfaces in [3].

Received January 15, 2019; accepted Februaty 06, 2019

2010 Mathematics Subject Classification. Primary 47S10; Secondary 53A04, 53B30 
Hamilton introduced a new algebra of the real quaternions. Additionally, he wanted to replicate what Gauss did with complex numbers and the Euclidean plane over the real numbers. So, Hamilton obtained the 4-dimensional real division algebra

$$
\mathcal{H}_{\mathbb{R}}=\left\{\mu_{1}+\mu_{2} \delta_{1}+\mu_{3} \delta_{2}+\mu_{4} \delta_{3} \mid \delta_{1}^{2}=\delta_{2}^{2}=\delta_{3}^{2}=\delta_{1} \delta_{2} \delta_{3}=-1\right\},
$$

where $\mu_{1}, \mu_{2}, \mu_{3}, \mu_{4} \in \mathbb{R}$. The key of Hamilton's work is the interpretation of the multiplication of the imaginary units as the wedge product of the canonical basis $\{\vec{i}, \vec{j}, \vec{k}\}$ of the 3 -dimensional Euclidean space, [4].

In next years, Cokle found new examples in [5]: coquaternions, tessarines and cotessarines. The first ones are precisely the split quaternions. Then, a split quaternion $\mu$ is a linear combination of the form

$$
\mathcal{P}_{\mathbb{R}}=\left\{\mu=\mu_{1}+\mu_{2} \delta_{1}+\mu_{3} \delta_{2}+\mu_{4} \delta_{3} \mid-\delta_{1}^{2}=\delta_{2}^{2}=\delta_{3}^{2}=\delta_{1} \delta_{2} \delta_{3}=1\right\},
$$

where $\mu_{1}, \mu_{2}, \mu_{3}, \mu_{4} \in \mathbb{R}$, [4]. In recent papers, split quaternions have been widely used . For example, Aslan and Kocakuşaklı studied canal surfaces with split quaternions in [1],[9]. Aslan gave quaternionic shape operator in [2], and Tunçer studied circular surfaces with split quaternions.

There are many studies on surfaces with quaternions and split quaternions considering the aforementioned articles, but construction of the Bäcklund transformation with split quaternions has not been studied until now. Because classical Bäcklund transformations mainly focus on the transformation of surfaces, the relationship between the results obtained with this study and the theory of surfaces can be investigated. Therefore, we will explain the relationship between Bäcklund Transformations of non-null curves and a timelike split quaternion having a timelike vector part, a timelike split quaternion having a spacelike vector part and a spacelike split quaternion.

\section{Preliminaries}

Let us recall some known concepts of split quaternion theory and Bishop frame given by [11] and [8].

Assume that $r=\left(r_{1}, r_{2}, r_{3}\right)$ and $s=\left(s_{1}, s_{2}, s_{3}\right)$ are two vectors in Minkowski 3 -space. Then, Lorentzian inner product and vector product of these curves are defined by

$$
\langle r, s\rangle_{L}=-r_{1} s_{1}+r_{2} s_{2}+r_{3} s_{3}
$$

and

$$
r \Lambda_{L} s=\left(r_{3} s_{2}-r_{2} s_{3}, r_{1} s_{3}-r_{3} s_{1}, r_{1} s_{2}-r_{2} s_{1}\right) .
$$

For a vector $r \in \mathbb{E}_{1}^{3}, r$ is called

i) a spacelike vector if $\langle r, r\rangle_{L}>0$ or $r=0$,

ii) a timelike vector if $\langle r, r\rangle_{L}<0$, 
iii) a null vector if $\langle r, r\rangle_{L}=0$ for $r \neq 0,[10]$.

Let $\mathcal{P}_{\mathbb{R}}$ denote a four dimensional vector space over a field $\mathbb{R}$ whose characteristics is greater than $2,[6]$ :

Split quaternion algebra is an associative, non-commutative non-division ring with four basic elements $\left\{1, \omega_{1}, \omega_{2}, \omega_{3}\right\}$ satisfying the equalities $-\omega_{1}^{2}=\omega_{2}^{2}=\omega_{3}^{2}=1$ and

$$
\omega_{1} * \omega_{2}=\omega_{3}, \omega_{2} * \omega_{3}=-\omega_{1}, \omega_{3} * \omega_{1}=\omega_{2} .
$$

Furthermore, $S_{w}=w_{1}$ and $\vec{V}_{w}=w_{2} \omega_{1}+w_{3} \omega_{2}+w_{4} \omega_{3}$ are scalar and vector parts of a real split quaternion $w=w_{1}+w_{2} \omega_{1}+w_{3} \omega_{2}+w_{4} \omega_{3}$, respectively. Let $w=$ $\left(w_{1}, w_{2}, w_{3}, w_{4}\right)$ and $q=\left(q_{1}, q_{2}, q_{3}, q_{4}\right)$ be two split quaternions. Then, the split quaternion product of the split quaternions $w$ and $q$ is defined as

$$
w * q=w_{1} q_{1}+\left\langle\vec{V}_{w}, \vec{V}_{q}\right\rangle_{L}+w_{1} \vec{V}_{q}+q_{1} \vec{V}_{w}+\vec{V}_{w} \Lambda_{L} \vec{V}_{q},
$$

where $\langle,\rangle_{L}$ and $\Lambda_{L}$ are Lorentzian inner product and vector product respectively. Also, a split quaternion $w=\left(w_{1}, w_{2}, w_{3}, w_{4}\right)$ is expressed as

i) $w$ is a spacelike if $-I_{w}=-w_{1}^{2}-w_{2}^{2}+w_{3}^{2}+w_{4}^{2}<0$,

ii) $w$ is a timelike if $-I_{w}=-w_{1}^{2}-w_{2}^{2}+w_{3}^{2}+w_{4}^{2}>0$,

iii) $w$ is a lightlike(null) quaternion if $-I_{w}=-w_{1}^{2}-w_{2}^{2}+w_{3}^{2}+w_{4}^{2}=0$. The norm of $w=\left(w_{1}, w_{2}, w_{3}, w_{4}\right)$ is defined as

$$
N_{w}=\sqrt{\left|w_{1}^{2}+w_{2}^{2}-w_{3}^{2}-w_{4}^{2}\right|} .
$$

If $N_{w}=1$ then $w$ is called unit split quaternion and $w_{0}=w / N_{w}$ is a unit split quaternion for $N_{w} \neq 0$.

Also, each spacelike unit split quaternion $w=\left(w_{1}, w_{2}, w_{3}, w_{4}\right)$ is expressed as

$$
w=N_{w}(\sinh \varphi+\varepsilon \cosh \varphi),
$$

where

$$
\sinh \varphi=\frac{w_{1}}{N_{w}}, \cosh \varphi=\frac{\sqrt{-w_{2}^{2}+w_{3}^{2}+w_{4}^{2}}}{N_{w}}
$$

and

$$
\varepsilon=\frac{w_{2} \omega_{1}+w_{3} \omega_{2}+w_{4} \omega_{3}}{\sqrt{-w_{2}^{2}+w_{3}^{2}+w_{4}^{2}}} .
$$

Each timelike split quaternion having a spacelike vector part $w=\left(w_{1}, w_{2}, w_{3}, w_{4}\right)$ is expressed as

$$
w=N_{w}(\cosh \varphi+\varepsilon \sinh \varphi)
$$

where

$$
\cosh \varphi=\frac{w_{1}}{N_{w}}, \sinh \varphi=\frac{\sqrt{-w_{2}^{2}+w_{3}^{2}+w_{4}^{2}}}{N_{w}}
$$


and

$$
\varepsilon=\frac{w_{2} \omega_{1}+w_{3} \omega_{2}+w_{4} \omega_{3}}{\sqrt{-w_{2}^{2}+w_{3}^{2}+w_{4}^{2}}} .
$$

Finally, each timelike split quaternion having a timelike vector part $w=\left(w_{1}, w_{2}, w_{3}, w_{4}\right)$ is expressed, as

$$
w=N_{w}(\cos \varphi+\varepsilon \sin \varphi),
$$

where

$$
\cos \varphi=\frac{w_{1}}{N_{w}}, \sin \varphi=\frac{\sqrt{w_{2}^{2}-w_{3}^{2}-w_{4}^{2}}}{N_{w}}
$$

and

$$
\varepsilon=\frac{w_{2} \omega_{1}+w_{3} \omega_{2}+w_{4} \omega_{3}}{\sqrt{w_{2}^{2}-w_{3}^{2}-w_{4}^{2}}}
$$

On the other hand, assume that $\alpha: s \rightarrow \alpha(s)$, which is parameterized by arclength parameter $s$, is a spatial curve in Minkowski 3-space. The relation between Bishop frame and Frenet frame of a curve $\alpha$ according to the arc-length parameter is governed by the relations:

$$
\begin{aligned}
\mathcal{T} & =\mathcal{T}, \\
\mathcal{N} & =\cos \theta \mathcal{N}_{1}-\sin \theta \mathcal{N}_{2}, \\
\mathcal{B} & =\sin \theta \mathcal{N}_{1}+\cos \theta \mathcal{N}_{2} .
\end{aligned}
$$

Also, $\kappa_{1}(s)=\kappa(s) \cos \theta(s)$ and $\kappa_{2}(s)=\tau(s) \sin \theta(s)$ are called Bishop curvatures.

The Bishop equations can be given as below, if the curve $\alpha$ is a timelike curve:

$$
\begin{aligned}
\mathcal{T}^{\prime} & =\kappa_{1} \mathcal{N}_{1}+\kappa_{2} \mathcal{N}_{2}, \\
\mathcal{N}_{1}^{\prime} & =\kappa_{1} \mathcal{T} \\
\mathcal{N}_{2}^{\prime} & =\kappa_{2} \mathcal{T}
\end{aligned}
$$

where

$$
\begin{gathered}
\kappa=\sqrt{\kappa_{1}^{2}+\kappa_{2}^{2}}, \theta=\arctan \left(\frac{\kappa_{2}}{\kappa_{1}}\right), \tau=\frac{d \theta}{d s} \\
\langle\mathcal{T}, \mathcal{T}\rangle_{L}=-1,\left\langle\mathcal{N}_{1}, \mathcal{N}_{1}\right\rangle_{L}=1,\left\langle\mathcal{N}_{2}, \mathcal{N}_{2}\right\rangle_{L}=1
\end{gathered}
$$

The Bishop equations can be given as below, if the curve $\alpha$ is a spacelike curve with a timelike principal normal:

$$
\begin{aligned}
\mathcal{T}^{\prime} & =\kappa_{1} \mathcal{N}_{1}-\kappa_{2} \mathcal{N}_{2}, \\
\mathcal{N}_{1}^{\prime} & =\kappa_{1} \mathcal{T}, \\
\mathcal{N}_{2}^{\prime} & =\kappa_{2} \mathcal{T}
\end{aligned}
$$


where

$$
\begin{aligned}
& \kappa=\sqrt{\left|\kappa_{2}^{2}-\kappa_{1}^{2}\right|}, \theta=\arg \tanh \left(\frac{\kappa_{2}}{\kappa_{1}}\right), \tau=\frac{d \theta}{d s} \\
& \langle\mathcal{T}, \mathcal{T}\rangle_{L}=1,\left\langle\mathcal{N}_{1}, \mathcal{N}_{1}\right\rangle_{L}=-1,\left\langle\mathcal{N}_{2}, \mathcal{N}_{2}\right\rangle_{L}=1
\end{aligned}
$$

The Bishop equations can be given as below, if the curve $\alpha$ is a spacelike curve with a timelike principal normal:

$$
\begin{aligned}
\mathcal{T}^{\prime} & =\kappa_{1} \mathcal{N}_{1}-\kappa_{2} \mathcal{N}_{2}, \\
\mathcal{N}_{1}^{\prime} & =-\kappa_{1} \mathcal{T} \\
\mathcal{N}_{2}^{\prime} & =-\kappa_{2} \mathcal{T}
\end{aligned}
$$

where

$$
\begin{gathered}
\kappa=\sqrt{\left|\kappa_{1}^{2}-\kappa_{2}^{2}\right|}, \theta=\arg \tanh \left(\frac{\kappa_{2}}{\kappa_{1}}\right), \tau=-\frac{d \theta}{d s} \\
\langle\mathcal{T}, \mathcal{T}\rangle_{L}=1,\left\langle\mathcal{N}_{1}, \mathcal{N}_{1}\right\rangle_{L}=1,\left\langle\mathcal{N}_{2}, \mathcal{N}_{2}\right\rangle_{L}=-1 .
\end{gathered}
$$

\section{Results And Discussion}

Let us start by assuming $q: I \rightarrow R \subset \mathcal{P}_{\mathbb{R}}$ is a unit split quaternion with arclength parameter $s,[7]$. Then, a split quaternion $q$ can be written as

$$
\begin{aligned}
q: I & \rightarrow \mathcal{P}_{\mathbb{R}} \\
s & \rightarrow q(s)=\sum_{i=1}^{4} q_{i}(s) \omega_{i} ; \quad 1 \leq i \leq 4, \omega_{1}=1 .
\end{aligned}
$$

\subsection{The Construction of Bäcklund Transformations of Timelike Curve}

Let us recall Bäcklund transformations of a timelike curve introduced by Karacan in [8] assume that $\alpha$ is a timelike curve and $\left\{\mathcal{T}, \mathcal{N}_{1}, \mathcal{N}_{2}\right\}$ and $\left\{\kappa_{1}, \kappa_{2}\right\}$ are its Bishop frame and Bishop curvatures, respectively. Then, the Bäcklund transformation of the curve $\alpha$ is expressed as

$$
\beta=\alpha+\frac{2 \mathcal{K} \tanh \gamma}{\kappa_{2}^{2}+\mathcal{K}^{2}}\left(\cosh \gamma \mathcal{T}+\sinh \gamma \mathcal{N}_{1}\right),
$$

where

$$
\mathcal{K}=\kappa_{2} \tan \frac{\phi}{2}
$$

and

$$
\frac{d \gamma}{d s}=\kappa_{2}^{\beta} \cosh \gamma \tan \frac{\phi}{2}-\kappa_{1}
$$


Theorem 3.1. Let $\beta$ be Bäcklund transformations of a timelike curve given by equation (3.3) in $\mathbb{E}_{1}^{3}$ and $q$ be a spacelike split quaternion defined as

$$
q=\sinh \gamma+\cosh \gamma \mathcal{N}_{1} .
$$

The Bäcklund transformations of a timelike curve with a spacelike split quaternion can be stated by

$$
\beta=\alpha+\frac{2 \mathcal{K} \tanh \gamma}{\kappa_{2}^{2}+\mathcal{K}^{2}}\left[\frac{1}{2}\left(q * \mathcal{N}_{2}-\mathcal{N}_{2} * q\right)+\mathcal{N}_{1} * q-\cosh \gamma\right] .
$$

Proof. Let us assume that $q$ is a spacelike split quaternion given in the form $q=$ $\sinh \gamma+\cosh \gamma \mathcal{N}_{1}$. As a direct result of equation (3.3), the equality

$$
\mathcal{N}_{1} * q=\sinh \gamma * \mathcal{N}_{1}+\cosh \gamma
$$

holds, which directs us to the equality

$$
\sinh \gamma * \mathcal{N}_{1}=\mathcal{N}_{1} * q-\cosh \gamma .
$$

By multiplying both sides of the equation (3.5) with $\mathcal{N}_{2}$ from the left side, we reach

$$
\mathcal{N}_{2} * q=\sinh \gamma * \mathcal{N}_{2}-\cosh \gamma \mathcal{T}
$$

In a similar way, the equation

$$
q * \mathcal{N}_{2}=\sinh \gamma * \mathcal{N}_{2}+\cosh \gamma \mathcal{T}
$$

can be obtained analoguously, hence both the equations of (3.9) and (3.10) allow us to write

$$
\cosh \gamma \mathcal{T}=\frac{1}{2}\left(q * \mathcal{N}_{2}-\mathcal{N}_{2} * q\right)
$$

As a consequence of the equations (3.8), (3.11), we immediately have the equation

$$
\cosh \gamma \mathcal{T}+\sinh \gamma \mathcal{N}_{1}=\frac{1}{2}\left(q * \mathcal{N}_{2}-\mathcal{N}_{2} * q\right)+\mathcal{N}_{1} * q-\cosh \gamma,
$$

which states the fact that Bäcklund transformations $\beta$ can be calculated as

$$
\beta=\alpha+\frac{2 \mathcal{K} \tanh \gamma}{\kappa_{2}^{2}+\mathcal{K}^{2}}\left[\frac{1}{2}\left(q * \mathcal{N}_{2}-\mathcal{N}_{2} * q\right)+\mathcal{N}_{1} * q-\cosh \gamma\right] .
$$

The above equalities direct us to the following theorem. 
Theorem 3.2. Let $\beta$ be Bäcklund transformations of a timelike curve given by equation (3.2) in $\mathbb{E}_{1}^{3}$ and $q$ be a spacelike split quaternion defined as

$$
q=\sinh \gamma+\cosh \gamma \mathcal{N}_{2}
$$

The Bäcklund transformations of a timelike curve with a spacelike split quaternion can be stated by

$$
\beta=\alpha+\frac{2 \mathcal{K} \tanh \gamma}{\kappa_{2}^{2}+\mathcal{K}^{2}}\left[\mathcal{N}_{1} * q\right]
$$

Proof. Assume that $q$ is a spacelike split quaternion defined as $q=\sinh \gamma+\cosh \gamma \mathcal{N}_{2}$. If we multiply both sides of the equation (3.14) with $\mathcal{N}_{1}$ from the left side, then

$$
\mathcal{N}_{1} * q=\cosh \gamma \mathcal{T}+\sinh \gamma \mathcal{N}_{1}
$$

In this case, the Bäcklund transformations $\beta$ can be calculated as

$$
\beta=\alpha+\frac{2 \mathcal{K} \tanh \gamma}{\kappa_{2}^{2}+\mathcal{K}^{2}}\left[\mathcal{N}_{1} * q\right] .
$$

The proof of the next theorem is similar to Theorem 1.

Theorem 3.3. Let $\beta$ be Bäcklund transformations of a timelike curve given by equation (3.2) in $\mathbb{E}_{1}^{3}$ and $q$ be a timelike split quaternion having a spacelike vector part defined as

$$
q=\cosh \gamma+\sinh \gamma \mathcal{N}_{2}
$$

The Bäcklund transformations of a timelike curve with a timelike split quaternion having a spacelike vector part can be stated by

$$
\beta=\alpha+\frac{2 \mathcal{K} \tanh \gamma}{\kappa_{2}^{2}+\mathcal{K}^{2}}[\mathcal{T} * q]
$$

Proposition 3.1. Let $q$ be a timelike split quaternion having a spacelike vector part defined as

$$
q=\cosh \gamma+\sinh \gamma \mathcal{N}_{2}
$$

and $u, v$ be any two vectors, where the angle between them is $\gamma$, and $\beta$ be Bäcklund transformations given by equation (3.19) in $\mathbb{E}_{1}^{3}$. Then, the Bäcklund transformations of a timelike curve with a timelike split quaternion having a spacelike vector part can be stated by

$$
\beta=\alpha+\frac{2 \mathcal{K} \tanh \gamma}{\kappa_{2}^{2}+\mathcal{K}^{2}}\left[\mathcal{T} * v * u^{-1}\right]
$$


where they satisfy the following conditions:

i) if $u$ and $v$ are timelike vectors, then $u$ and $v$ are perpendicular to $\mathcal{N}_{2}$,

ii) if $u$ and $v$ are spacelike vectors, then $|\langle u, v\rangle|>1$ and $u$ and $v$ are perpendicular to $\mathcal{N}_{2}$.

Theorem 3.4. Let $\beta$ be Bäcklund transformations of a timelike curve given by the equation (3.2) in $\mathbb{E}_{1}^{3}$ and $q$ be a timelike split quaternion having a spacelike vector part defined as

$$
q=\cosh \gamma+\sinh \gamma \mathcal{N}_{1} .
$$

The Bäcklund transformations of a timelike split quaternion having a spacelike vector part can be stated by

$$
\beta=\alpha+\frac{2 \mathcal{K} \tanh \gamma}{\kappa_{2}^{2}+\mathcal{K}^{2}}\left[\frac{1}{2}(\mathcal{T} * q+q * \mathcal{T})+q-\cosh \gamma\right] .
$$

Proof. Let us assume that $q$ is a timelike split quaternion having a spacelike vector part given in the form $\cosh \gamma+\sinh \gamma \mathcal{N}_{1}$. As a direct result of the equation (3.22), the equality

$$
\sinh \gamma \mathcal{N}_{1}=q-\cosh \gamma
$$

holds, which directs us to the equality. By multiplying both sides of the equation (3.22) with $\mathcal{T}$ from the left side, we reach

$$
\mathcal{T} * q=\cosh \mathcal{T}-\sinh \gamma \mathcal{N}_{2} .
$$

In a similar way, the equation

$$
q * \mathcal{T}=\cosh \mathcal{T}+\sinh \gamma \mathcal{N}_{2}
$$

can be obtained analoguously, hence both the equations of (3.25) and (3.26) allow us to write

$$
\cosh \gamma \mathcal{T}=\frac{1}{2}(\mathcal{T} * q+q * \mathcal{T}) .
$$

As a consequence of the equations (3.24), (3.27), we immediately have the equation

$$
\cosh \gamma \mathcal{T}+\sinh \gamma \mathcal{N}_{1}=\frac{1}{2}(\mathcal{T} * q+q * \mathcal{T})+q-\cosh \gamma
$$

In this case, the Bäcklund transformations with a timelike split quaternion having a spacelike vector part can be calculated as

$$
\beta=\alpha+\frac{2 \mathcal{K} \tanh \gamma}{\kappa_{2}^{2}+\mathcal{K}^{2}}\left[\frac{1}{2}(\mathcal{T} * q+q * \mathcal{T})+q-\cosh \gamma\right]
$$


Proposition 3.2. Let $q$ be a timelike split quaternion having a spacelike vector part defined as

$$
q=\cosh \gamma+\sinh \gamma \mathcal{N}_{1}
$$

and $u, v$ be any two vectors, where the angle between them is $\gamma$, and $\beta$ be Bäcklund transformations given by the equation (3.23) in $\mathbb{E}_{1}^{3}$. Then, the Bäcklund transformations of a timelike curve with a timelike split quaternion having a spacelike vector part can be stated by

$$
\beta=\alpha+\frac{2 \mathcal{K} \tanh \gamma}{\kappa_{2}^{2}+\mathcal{K}^{2}}\left[\frac{1}{2}\left(\mathcal{T} * v * u^{-1}+v * u^{-1} * \mathcal{T}\right)+v * u^{-1}-\cosh \gamma\right],
$$

where they satisfy the following conditions;

i) if $u$ and $v$ are timelike vectors, then $u$ and $v$ are perpendicular to $\mathcal{N}_{1}$,

ii) if $u$ and $v$ are spacelike vectors, then $|\langle u, v\rangle|>1$ and $u$ and $v$ are perpendicular to $\mathcal{N}_{1}$.

In the light of the above theorems, the following theorems will be given without proof.

\subsection{The Construction of Bäcklund Transformations of Spacelike Curve with Spacelike Principal Normal}

Let us recall Bäcklund transformations of a spacelike curve with spacelike principal normal introduced by Karacan in [8]. Therefore, we assume that $\alpha$ is a spacelike curve with a spacelike principal normal and $\left\{\mathcal{T}, \mathcal{N}_{1}, \mathcal{N}_{2}\right\}$ and $\left\{\kappa_{1}, \kappa_{2}\right\}$ are its Bishop frame and Bishop curvatures, respectively. Then, the Bäcklund transformation of the curve $\alpha$ is expressed as

$$
\beta=\alpha+\frac{2 \mathcal{K} \tanh \gamma}{\kappa_{2}^{2}-\mathcal{K}^{2}}\left(\cos \gamma \mathcal{T}+\sin \gamma \mathcal{N}_{1}\right),
$$

where

$$
\mathcal{K}=\kappa_{2} \tan \frac{\phi}{2}
$$

and

$$
\frac{d \gamma}{d s}=-\kappa_{2} \cos \gamma \tanh \frac{\phi}{2}-\kappa_{1}^{\beta} .
$$

Theorem 3.5. Let $\beta$ be Bäcklund transformations of a spacelike curve with spacelike principal normal given by the equation (3.32) in $\mathbb{E}_{1}^{3}$ and $q$ be a timelike split quaternion having a timelike vector part defined as

$$
q=\cos \gamma+\sin \gamma \mathcal{N}_{2}
$$


The Bäcklund transformations of a spacelike curve having spacelike principal normal with a timelike split quaternion having a timelike vector part can be stated by

$$
\beta=\alpha+\frac{2 \mathcal{K} \tanh \gamma}{\kappa_{2}^{2}-\mathcal{K}^{2}}[\mathcal{T} * q] .
$$

Proposition 3.3. Let $q$ be a timelike split quaternion having a timelike vector part defined as

$$
q=\cos \gamma+\sin \gamma \mathcal{N}_{2}
$$

and $u, v$ be any two spacelike vectors, when the angle between them is $\gamma$, and $\beta$ be Bäcklund transformations given by equation (3.36) in $\mathbb{E}_{1}^{3}$. Then, Bäcklund transformations of a spacelike curve having a spacelike principal normal with a timelike split quaternion having a timelike vector part can be stated by

$$
\beta=\alpha+\frac{2 \mathcal{K} \tanh \gamma}{\kappa_{2}^{2}-\mathcal{K}^{2}}[\mathcal{T} * u * v],
$$

where $u$ and $v$ are perpendicular to $\mathcal{N}_{2}$.

\subsection{The Construction of Bäcklund Transformations of Spacelike Curve with Spacelike Binormal}

Let us recall Bäcklund transformations of a spacelike curve with spacelike binormal introduced by Karacan in [8]. Hence, we assume that $\alpha$ is a spacelike curve with spacelike binormal and $\left\{\mathcal{T}, \mathcal{N}_{1}, \mathcal{N}_{2}\right\}$ and $\left\{\kappa_{1}, \kappa_{2}\right\}$ are its Bishop frame and Bishop curvatures, respectively. Then, the Bäcklund transformation of the curve $\alpha$ is expressed as

$$
\beta=\alpha+\frac{2 \mathcal{K} \sinh \gamma}{\kappa_{2}^{2}-\mathcal{K}^{2}}\left(\cosh \gamma \mathcal{T}+\sinh \gamma \mathcal{N}_{1}\right),
$$

where

$$
\mathcal{K}=\kappa_{2} \tanh \frac{\phi}{2}
$$

and

$$
\frac{d \gamma}{d s}=-\kappa_{2} \cosh \gamma \tanh \frac{\phi}{2}-\kappa_{1}^{\beta} .
$$

Theorem 3.6. Let $\beta$ be Bäcklund transformations of a spacelike curve with spacelike binormal given by equation (3.41) in $\mathbb{E}_{1}^{3}$ and $q$ be a spacelike split quaternion defined as

$$
q=\sinh \gamma+\cosh \gamma \mathcal{N}_{2} .
$$


The Bäcklund transformations of a spacelike curve with spacelike binormal with a spacelike split quaternion can be stated by

$$
\beta=\alpha+\frac{2 \mathcal{K} \sinh \gamma}{\kappa_{2}^{2}-\mathcal{K}^{2}}\left[q * \mathcal{N}_{1}\right]
$$

Theorem 3.7. Let $\beta$ be Bäcklund transformations of a spacelike curve with a spacelike binormal given by the equation (3.39) in $\mathbb{E}_{1}^{3}$ and $q$ be a spacelike split quaternion defined as

$$
q=\sinh \gamma+\cosh \gamma \mathcal{T}
$$

The Bäcklund transformations of a spacelike curve having a spacelike binormal with a spacelike split quaternion can be stated by

$$
\beta=\alpha+\frac{2 \mathcal{K} \sinh \gamma}{\kappa_{2}^{2}-\mathcal{K}^{2}}\left[q-\sinh \gamma+\frac{1}{2}\left(\mathcal{N}_{1} * q+q * \mathcal{N}_{1}\right)\right]
$$

Theorem 3.8. Let $\beta$ be Bäcklund transformations of a spacelike curve with spacelike binormal given by equation (3.39) in $\mathbb{E}_{1}^{3}$ and $q$ be a timelike split quaternion having a spacelike vector part defined as

$$
q=\cosh \gamma+\sinh \gamma \mathcal{T} .
$$

The Bäcklund transformations of a spacelike curve having a spacelike binormal with a timelike split quaternion having a spacelike vector part can be stated by

$$
\beta=\alpha+\frac{2 \mathcal{K} \sinh \gamma}{\kappa_{2}^{2}-\mathcal{K}^{2}}\left[q * \mathcal{T}-\sinh \gamma+\frac{1}{2}\left(\mathcal{N}_{2} * q-q * \mathcal{N}_{2}\right)\right]
$$

Proposition 3.4. Let $q$ be a timelike split quaternion having a spacelike vector part defined as

$$
q=\cosh \gamma+\sinh \gamma \mathcal{T}
$$

and $u, v$ be any two vectors, when the angle between them is $\gamma$, and $\beta$ be Bäcklund transformations given by the equation (3.47) in $\mathbb{E}_{1}^{3}$. Then, Bäcklund transformations of a spacelike curve having a spacelike binormal with a timelike split quaternion having a spacelike vector part can be stated by

$$
\beta=\alpha+\frac{2 \mathcal{K} \sinh \gamma}{\kappa_{2}^{2}-\mathcal{K}^{2}}\left[v * u^{-1} * \mathcal{T}-\sinh \gamma+\frac{1}{2}\left(\mathcal{N}_{2} * v * u^{-1}-v * u^{-1} * \mathcal{N}_{2}\right)\right]
$$

where they satisfy the following conditions:

i) if $u$ and $v$ are timelike vectors, then $u$ and $v$ are perpendicular to $\mathcal{T}$,

ii) if $u$ and $v$ are spacelike vectors, then $|\langle u, v\rangle|>1$ and $u$ and $v$ are perpendicular to $\mathcal{T}$. 
Theorem 3.9. Let $\beta$ be Bäcklund transformations of a spacelike curve with a spacelike binormal given by the equation (3.39) in $\mathbb{E}_{1}^{3}$ and $q$ be a timelike split quaternion having a spacelike vector part defined as

$$
q=\cosh \gamma+\sinh \gamma \mathcal{N}_{2} .
$$

The Bäcklund transformations of a spacelike curve having a spacelike binormal with a timelike split quaternion having a spacelike vector part can be stated by

$$
\beta=\alpha+\frac{2 \mathcal{K} \sinh \gamma}{\kappa_{2}^{2}-\mathcal{K}^{2}}[\mathcal{T} * q] .
$$

Proposition 3.5. Let $q$ be a timelike split quaternion having a spacelike vector part defined as

$$
q=\cosh \gamma+\sinh \gamma \mathcal{N}_{2} .
$$

and $u, v$ be any two vectors, when the angle between them is $\gamma$, and $\beta$ be Bäcklund transformations given by the equation (3.51) in $\mathbb{E}_{1}^{3}$. Then, Bäcklund transformations of a spacelike curve having a spacelike binormal with a timelike split quaternion having a spacelike vector part can be stated by

$$
\beta=\alpha+\frac{2 \mathcal{K} \sinh \gamma}{\kappa_{2}^{2}-\mathcal{K}^{2}}\left[\mathcal{T} * v * u^{-1}\right],
$$

where they satisfy the following conditions;

i) if $u$ and $v$ are timelike vectors, then $u$ and $v$ are perpendicular to $\mathcal{N}_{2}$,

ii) if $u$ and $v$ are spacelike vectors, then $|\langle u, v\rangle|>1$ and $u$ and $v$ are perpendicular to $\mathcal{N}_{2}$.

\section{REFEREN CES}

1. S. Aslan \& Y.YaYLI: Canal Surfaces in Minkowski 3-Space. International Journal of Geometry. 5(2) (2016), 142-149.

2. S. Aslan \& Y. Yayli: Quaternionic Shape Operator. Advances in Applied Clifford Algebras 27(4) (2017), 2921-2931.

3. P. B̈̈сK: Bäcklund Transformations for Minimal Surfaces. Linkoping University, Department of Mathematics, MSc. Thesis

4. A. Cigliola: Split quaternions, generalized quaternions and integer-valued polynomials. Universit a Degli Studi Roma Tre, PhD Thesis in Mathematics. (2012).

5. J. CocKLE: On systems of algebra involving more than one imaginary; and on equations of the fifth degree. The London, Edinburgh, and Dublin Philosophical Magazine and Journal of Science. 35(238) (1849), 434-437.

6. I. Gok, O. Z. OkuYucu, F. Kahraman, H.H. Hacisalihoglu: On the quaternionic B 2-slant helices in the Euclidean space E 4. Advances in Applied Clifford Algebras. 21(4) (2011), 707-719. 
7. M. A. Gungor, T. ERISIR: On the Quaternionic Curves in the Semi-Euclidean Space $\mathbb{E}_{2}^{4}$. Caspian Journal of Mathematical Sciences (CJMS). 7(1) (2018), 36-45.

8. M.K. KARACAN, Y. TUNCER: Bäcklund transformations according to Bishop frame in E3/1. Acta et Commentationes Universitatis Tartuensis de Mathematica. 19(2) (2015), 75-85.

9. E. Kochkusakli, , O.O. Tuncer, I. Gok, Y. Yayli: A new representation of canal surfaces with split quaternions in Minkowski 3-space. Advances in Applied Clifford Algebras. 27(2) (2017), 1387-1409.

10. B. O’NeILl: Semi-Riemannian Geometry. In Academic Press; New York, (1983).

11. M. OzDEmiR: The roots of a split quaternion. Applied Mathematics Letters. 22(2) (2009), 258-263.

12. D.C. Sen, A.R. Chowdhury: On Darboux Bäcklund Transformation and Position Type Solution of Coupled Nonlinear Optical Waves. Acta Phy. Pol. 1-2(98) (2000).

13. O.O. Tuncer, Z. Canakci, I. Gok, Y. Yayli: Circular Surfaces with Split Quaternionic Representations in Minkowski 3-space. Advances in Applied Clifford Algebras. 28(3) (2018), 63.

14. J. WeIss: Bäcklund Transformations, Focal Surfaces and The Two-dimensional. Toda lattice. 7-8(137) (1989), 365-368.

15. E. B. SAfF and R. S. VArga: On incomplete polynomials II. Pacific J. Math. 92 (1981), 161-172.

16. HTTPS://NPTEL.AC.IN/COURSES/112105165/LEC38.PDF.

Muhammed Talat Sariaydin

Faculty of Science

Department of Mathematics

42130 Konya, Turkey

talatsariaydin@gmail.com 